Proceedings of the Edinburgh Mathematical Society (2008) 51, 285-296 (C)

DOI:10.1017/S0013091505001574 Printed in the United Kingdom

\title{
UNBOUNDED B-FREDHOLM OPERATORS ON HILBERT SPACES
}

\author{
M. BERKANI ${ }^{1}$ AND N. CASTRO-GONZÁLEZ ${ }^{2}$ \\ ${ }^{1}$ Département de Mathématiques, Faculté des Sciences, \\ Université Mohammed I, Oujda, Morocco \\ (berkani@sciences.univ-oujda.ac.ma) \\ ${ }^{2}$ Facultad de Informática, Universidad Politécnica de Madrid, \\ 28660 Madrid, Spain (nieves@fi.upm.es)
}

(Received 10 November 2005)

\begin{abstract}
This paper is concerned with the study of a class of closed linear operators densely defined on a Hilbert space $H$ and called B-Fredholm operators. We characterize a B-Fredholm operator as the direct sum of a Fredholm closed operator and a bounded nilpotent operator. The notion of an index of a B-Fredholm operator is introduced and a characterization of B-Fredholm operators with index 0 is given in terms of the sum of a Drazin closed operator and a finite-rank operator. We analyse the properties of the powers $T^{m}$ of a closed B-Fredholm operator and we establish a spectral mapping theorem.
\end{abstract}

Keywords: unbounded B-Fredholm operators; index; Drazin inverse

2000 Mathematics subject classification: Primary 47A53; 47A55

\section{Introduction}

By $\mathcal{C}(H)$ we denote the set of all linear closed operators from $H$ to $H$, where $H$ is a Hilbert space. We write $\mathcal{D}(T), \mathcal{N}(T)$ and $\mathcal{R}(T)$ for the domain, nullspace and range of an operator $T \in \mathcal{C}(H)$. An operator $T \in \mathcal{C}(H)$ is called a Fredholm operator if both the nullity of $T, n(T)=\operatorname{dim} \mathcal{N}(T)$, and the defect of $T, d(T)=\operatorname{codim} \mathcal{R}(T)$, are finite. The index $\kappa(T)$ of a Fredholm operator $T$ is defined by $\kappa(T)=n(T)-d(T)$. It is well known that if $T$ is Fredholm, then $\mathcal{R}(T)$ is closed. The class of quasi-Fredholm operators on a Hilbert space was first studied by Labrousse [8]. Most of the results given therein extend easily to the Banach space case.

Definition 1.1. Let $T \in \mathcal{C}(H)$ and let

$$
\Delta(T)=\left\{n \in \mathbb{N}: \forall m \in \mathbb{N}, m \geqslant n \Rightarrow \mathcal{R}\left(T^{n}\right) \cap \mathcal{N}(T) \subseteq \mathcal{R}\left(T^{m}\right) \cap \mathcal{N}(T)\right\}
$$

Then the degree of stable iteration of $T$ is defined as $\operatorname{dis}(T)=\inf \Delta(T)($ with $\operatorname{dis}(T)=\infty$ if $\Delta(T)=\emptyset)$. 
Definition 1.2. Let $T \in \mathcal{C}(H)$ and let $d \in \mathbb{N}$. Then $T$ is quasi-Fredholm of degree $d$ if and only if the following three conditions hold:

(i) $\operatorname{dis}(T)=d$;

(ii) $\mathcal{R}\left(T^{d}\right) \cap \mathcal{N}(T)$ is closed in $H$;

(iii) $\mathcal{R}(T)+\mathcal{N}\left(T^{d}\right)$ is closed in $H$.

By q- $\Phi(d)$ we denote the set of all quasi-Fredholm operators of degree $d$. The class of B-Fredholm bounded linear operators acting on a Banach space was studied first by Berkani in [1] (and further in [2, 3]). Given a bounded linear operator $T$, for each integer $n$, define $T_{n}$ as the restriction of $T$ to $\mathcal{R}\left(T^{n}\right)$ viewed as a map from $\mathcal{R}\left(T^{n}\right)$ into $\mathcal{R}\left(T^{n}\right)$. Then $T$ is called a B-Fredholm operator if, for some integer $n$, the range space $\mathcal{R}\left(T^{n}\right)$ is closed and $T_{n}$ is a Fredholm operator. The index of a B-Fredholm operator $T$ is defined as the index of the Fredholm operator $T_{n}$. In [1] , it was shown that a bounded operator $T$ is a B-Fredholm operator if and only if there exists an integer $d \in \mathbb{N}$ such that $T \in \mathrm{q}-\Phi(d)$ and such that $\mathcal{N}(T) \cap \mathcal{R}\left(T^{d}\right)$ is of finite dimension and $\mathcal{R}(T)+\mathcal{N}\left(T^{d}\right)$ is of finite codimension. Moreover, if $T$ is B-Fredholm then $\operatorname{ind}(T)=$ $\operatorname{dim} \mathcal{N}(T) \cap \mathcal{R}\left(T^{d}\right)-\operatorname{codim} \mathcal{R}(T)+\mathcal{N}\left(T^{d}\right)$. Based on this characterization of B-Fredholm bounded operators, we introduce the class of B-Fredholm closed linear operators acting on a Hilbert space $H$ and study its properties. Mainly, we prove that an operator $T \in \mathcal{C}(H)$ densely defined on $H$ is a B-Fredholm operator if and only if $T=T_{0} \oplus T_{1}$, where $T_{0}$ is a closed Fredholm operator and $T_{1}$ is a nilpotent operator. Associated with a B-Fredholm operator we introduce the notion of index and we characterize B-Fredholm operators of index 0 in terms of the sum of a Drazin closed operator and a finite-rank operator.

Finally, we prove that the powers $T^{m}$ of a closed B-Fredholm operator are closed B-Fredholm operators and their indices are related by $\operatorname{ind}\left(T^{m}\right)=m \cdot \operatorname{ind}(T)$ and we establish a spectral mapping theorem. These results are an extension of similar results obtained in $[\mathbf{1}, \mathbf{2}]$ for the class of closed Fredholm operators acting on a Hilbert space.

\section{Definition and characterizations of the B-Fredholm closed operators}

In this section we define the set of B-Fredholm operators and we investigate its properties.

Definition 2.1. Let $T \in \mathcal{C}(H)$ be densely defined on $H$. Then $T$ is called a B-Fredholm operator if there exists an integer $d \in \Delta(T)$ such that the following conditions are satisfied:

(i) $\operatorname{dim} \mathcal{N}(T) \cap \mathcal{R}\left(T^{d}\right)<\infty$;

(ii) $\operatorname{codim} \mathcal{R}(T)+\mathcal{N}\left(T^{d}\right)<\infty$.

In this case, the index of $T$ is defined as the number

$$
\operatorname{ind}(T)=\operatorname{dim} \mathcal{N}(T) \cap \mathcal{R}\left(T^{d}\right)-\operatorname{codim} \mathcal{R}(T)+\mathcal{N}\left(T^{d}\right) .
$$


From [4, Lemma 2.3] it follows that each B-Fredholm operator is a quasi-Fredholm operator of degree $d_{0}=\operatorname{dis}(T)$. We also observe that the definition of the index of a BFredholm operator is independent of the integer $d \in \Delta(T)$ chosen. Indeed, if $d_{0}=\operatorname{dis}(T)$ and we take any $d \in \Delta(T)$, then $\mathcal{N}(T) \cap \mathcal{R}\left(T^{d_{0}}\right)=\mathcal{N}(T) \cap \mathcal{R}\left(T^{d}\right)$. On the other hand, we have that $d_{0} \in \Delta(T)$. Hence, by $\left[8\right.$, Proposition 3.1.1] it follows that $\mathcal{R}(T)+\mathcal{N}\left(T^{d}\right) \subseteq$ $\mathcal{R}(T)+\mathcal{N}\left(T^{d_{0}}\right)$ and, consequently, $\mathcal{R}(T)+\mathcal{N}\left(T^{d}\right)=\mathcal{R}(T)+\mathcal{N}\left(T^{d_{0}}\right)$ because $d \geqslant d_{0}$.

Let $\mathrm{BF}(H)$ be the class of all B-Fredholm closed operators densely defined on the Hilbert space $H$.

Remark 2.2. Assume that $T \in \mathcal{C}(H)$ is a B-Fredholm operator densely defined on $H$, and let $d=\operatorname{dis}(T)$. Then $\mathcal{N}(T) \cap \mathcal{R}\left(T^{d}\right)$ is of finite dimension and $\mathcal{R}(T)+\mathcal{N}\left(T^{d}\right)$ is of finite codimension. From [8, Corollary 3.3.1], we know that $\mathcal{R}\left(T^{n}\right)$ is closed for all $n \geqslant d$. Consider the restriction of $T$ to $\mathcal{R}\left(T^{d}\right), T_{d}: \mathcal{R}\left(T^{d}\right) \rightarrow \mathcal{R}\left(T^{d}\right)$. We have that $\mathcal{D}\left(T_{d}\right)=\mathcal{D}(T) \cap \mathcal{R}\left(T^{d}\right)$ and

$$
\operatorname{dim} \mathcal{N}\left(T_{d}\right)=\operatorname{dim} \mathcal{N}(T) \cap \mathcal{R}\left(T^{d}\right)<\infty
$$

On the other hand, we have $\mathcal{R}\left(T_{d}\right)=\mathcal{R}\left(T^{d+1}\right)$ and, by [6, Lemma 3.2],

$$
\frac{\mathcal{R}\left(T^{d}\right)}{\mathcal{R}\left(T^{d+1}\right)} \cong \frac{\mathcal{D}\left(T^{d}\right)}{\left(\mathcal{R}(T)+\mathcal{N}\left(T^{d}\right)\right) \cap \mathcal{D}\left(T^{d}\right)} \cong \frac{\mathcal{D}\left(T^{d}\right)+\mathcal{R}(T)+\mathcal{N}\left(T^{d}\right)}{\mathcal{R}(T)+\mathcal{N}\left(T^{d}\right)}
$$

Hence, we get

$$
\operatorname{dim} \frac{\mathcal{R}\left(T^{d}\right)}{\mathcal{R}\left(T^{d+1}\right)}=\operatorname{dim} \frac{\mathcal{D}\left(T^{d}\right)}{\left(\mathcal{R}(T)+\mathcal{N}\left(T^{d}\right)\right) \cap \mathcal{D}\left(T^{d}\right)} \leqslant \operatorname{dim} \frac{H}{\mathcal{R}(T)+\mathcal{N}\left(T^{d}\right)}<\infty
$$

Hence, we have proved that $T_{d}$ is a Fredholm closed operator. However, the fact that, for some integer $n$, the range space $\mathcal{R}\left(T^{n}\right)$ is closed and the restriction operator $T_{n}$ is a Fredholm closed operator does not allow us to conclude that $T$ is a B-Fredholm operator in the sense of Definition 2.1, as in the case of bounded operators [4].

We observe that if $T$ is densely defined, then the adjoint operator $T^{*}$ exists, belongs to $\mathcal{C}\left(H^{*}\right)$ and is densely defined.

Proposition 2.3. Let $T \in \mathcal{C}(H)$ be densely defined. If $T$ is a B-Fredholm, then $T^{*}$ is $B$-Fredholm and ind $\left(T^{*}\right)=-\operatorname{ind}(T)$.

Proof. Assume that $T$ is B-Fredholm and let $d=\operatorname{dis}(T)$. Since $T \in \mathrm{q}-\Phi(d)$, from $[\mathbf{8}$, Proposition 3.3.5] it follows that $T^{*} \in \mathrm{q}-\Phi(d)$ and

$$
\mathcal{R}(T)+\mathcal{N}\left(T^{d}\right)=\left(\mathcal{N}\left(T^{*}\right) \cap \mathcal{R}\left(T^{* d}\right)\right)^{\perp}, \quad \mathcal{R}\left(T^{*}\right)+\mathcal{N}\left(T^{* d}\right)=\left(\mathcal{N}(T) \cap \mathcal{R}\left(T^{d}\right)\right)^{\perp} .
$$

Hence, we get

$$
\begin{aligned}
\operatorname{ind}\left(T^{*}\right) & =\operatorname{dim} \mathcal{R}\left(T^{* d}\right) \cap \mathcal{N}\left(T^{*}\right)-\operatorname{codim} \mathcal{R}\left(T^{*}\right)+\mathcal{N}\left(T^{* d}\right) \\
& =\operatorname{codim} \mathcal{R}(T)+\mathcal{N}\left(T^{d}\right)-\operatorname{dim} \mathcal{R}\left(T^{d}\right) \cap \mathcal{N}(T)=-\operatorname{ind}(T) .
\end{aligned}
$$


Now we give a characterization of B-Fredholm closed operators based on the Kato decomposition of quasi-Fredholm operators defined in [8].

Theorem 2.4. Let $T \in \mathcal{C}(H)$ with $\mathcal{D}(T)$ dense in $H$. Then $T$ is B-Fredholm with $d=\operatorname{dis}(T)$ if and only if there exists two closed subspaces $M$ and $N$ of $H$ such that

(a) $H=M \oplus N, T(\mathcal{D}(T) \cap M) \subseteq M, N \subseteq \mathcal{N}\left(T^{d}\right)$ and $N \nsubseteq \mathcal{N}\left(T^{d-1}\right)$;

(b) if $T_{0}=\left.T\right|_{M}$, then $T_{0}$ is a closed Fredholm operator densely defined on $M$ to $M$;

(c) if $T_{1}=\left.T\right|_{N}$, then $T_{1}$ is nilpotent of degree $d$.

In this case $\operatorname{ind}(T)=\kappa\left(T_{0}\right)$.

Proof. Assume that $T$ is B-Fredholm, that is, $T \in \mathrm{q}-\Phi(d), \operatorname{dim}\left(\mathcal{N}(T) \cap \mathcal{R}\left(T^{d}\right)\right)<\infty$ and $\operatorname{codim}\left(\mathcal{R}(T)+\mathcal{N}\left(T^{d}\right)\right)<\infty$.

By [8, Theorem 3.2.1], there exist two closed subspaces $M$ and $N$ such that conditions (a) and (c) given in Theorem 2.4 are fulfilled and also the following condition is satisfied:

( $\tilde{b}) T_{0}$ is a closed operator on $M$ to $M, \mathcal{R}\left(T_{0}\right)$ is closed in $M$ and $\operatorname{dis}\left(T_{0}\right)=0$.

We observe that $T_{0}$ is densely defined on $M$ because $T$ is densely defined on $H$. Let us prove that $T_{0}$ is Fredholm and $\operatorname{ind}(T)=\operatorname{ind}\left(T_{0}\right)$. From the proof of [8, Theorem 3.2.1, Equations (3.2.22) and (3.2.23)] we get

$$
\mathcal{R}\left(T_{0}\right) \oplus N=\mathcal{R}(T)+\mathcal{N}\left(T^{d}\right) \quad \text { and } \quad \mathcal{N}\left(T_{0}\right)=\mathcal{N}(T) \cap \mathcal{R}\left(T^{d}\right) .
$$

Hence, we obtain $\operatorname{dim} \mathcal{N}\left(T_{0}\right)=\operatorname{dim} \mathcal{N}(T) \cap \mathcal{R}\left(T^{d}\right)<\infty$.

On the other hand, since $\operatorname{codim} \mathcal{R}(T)+\mathcal{N}\left(T^{d}\right)<\infty$, we have $H=L \oplus \mathcal{R}(T)+\mathcal{N}\left(T^{d}\right)$, where $L$ is a finite-dimensional subspace. Now, let $P_{M}: X \rightarrow M$ be the linear projection onto $M$ along $N$. Then, using the first relation in (2.1), we find that $M=P_{M}(L)+\mathcal{R}\left(T_{0}\right)$ and, consequently, $\mathcal{R}\left(T_{0}\right)$ is of finite codimension in $M$. Thus, we have proved that $T_{0}$ is Fredholm. Finally, we can write $M=K \oplus \mathcal{R}\left(T_{0}\right)$, where $K$ is a finite-dimensional subspace of $M$. Therefore, $H=K \oplus\left(\mathcal{R}\left(T_{0}\right) \oplus N\right)=K \oplus\left(\mathcal{R}(T)+\mathcal{N}\left(T^{d}\right)\right)$ and, hence, we conclude that the codimension of $\mathcal{R}(T)+\mathcal{N}\left(T^{d}\right)$ in $H$ coincides with the codimension of $\mathcal{R}\left(T_{0}\right)$ in $M$. Therefore, $\operatorname{ind}(T)=\kappa\left(T_{0}\right)$.

Conversely, assume that there exist two closed subspaces $M$ and $N$ satisfying conditions (a)-(c). By [8, Theorem 3.2.2], and the proof of this result, we find that $T \in \mathrm{q}-\Phi(d)$ and the relations (2.1) given in the present theorem are verified. Hence, we obtain

$$
\operatorname{dim} \mathcal{N}(T) \cap \mathcal{R}\left(T^{d}\right)=\operatorname{dim} \mathcal{N}\left(T_{0}\right)<\infty
$$

and

$$
\operatorname{dim} \frac{H}{\mathcal{R}(T)+\mathcal{N}\left(T^{d}\right)}=\operatorname{dim} \frac{M \oplus N}{\mathcal{R}\left(T_{0}\right) \oplus N}=\operatorname{dim} \frac{M}{\mathcal{R}\left(T_{0}\right)}<\infty .
$$

Consequently, $T$ is B-Fredholm and $\operatorname{ind}(T)=\kappa\left(T_{0}\right)$. 
Next, we consider the perturbation of a closed B-Fredholm operator by a finite-rank operator. Recall that a linear bounded operator $F$ is called a finite-rank operator if $\operatorname{dim} \mathcal{R}(F)<\infty$.

Proposition 2.5. Let $T \in \mathcal{C}(H)$ be a B-Fredholm operator with $\mathcal{D}(T)$ dense in $H$ and let $F \in \mathcal{B}(H)$ be a finite-rank operator. Then $T+F$ is a B-Fredholm operator and $\operatorname{ind}(T+F)=\operatorname{ind}(T)$.

Proof. Let $d=\operatorname{dis}(T)$. We observe that, by [9, Theorem 15],

$$
\mathcal{N}(T+F) \cap \mathcal{R}\left((T+F)^{d}\right)=\mathcal{N}(T) \cap \mathcal{R}\left(T^{d}\right)+S
$$

and

$$
\mathcal{N}\left(T^{*}+F^{*}\right) \cap \mathcal{R}\left((T+F)^{* d}\right)=\mathcal{N}\left(T^{*}\right) \cap \mathcal{R}\left(T^{* d}\right)+G,
$$

where $S$ and $G$ are finite-dimensional subspaces of $H$ and $H^{*}$, respectively. Hence, we conclude that $\operatorname{dim} \mathcal{N}(T+F) \cap \mathcal{R}\left((T+F)^{d}\right) \leqslant \operatorname{dim} \mathcal{N}(T) \cap \mathcal{R}\left(T^{d}\right)+\operatorname{dim} S<\infty$ and

$$
\begin{aligned}
\operatorname{codim} \mathcal{R}(T)+\mathcal{N}\left(T^{d}\right) & =\operatorname{dim} \mathcal{N}\left(T^{*}+F^{*}\right) \cap \mathcal{R}\left((T+F)^{* d}\right) \\
& \leqslant \operatorname{dim} \mathcal{N}\left(T^{*}\right) \cap \mathcal{R}\left(T^{* d}\right)+\operatorname{dim} G<\infty .
\end{aligned}
$$

Therefore, $T+F$ is B-Fredholm.

By Theorem 2.4, relative to the space decomposition $H=M \oplus N$ we have $T=T_{0} \oplus T_{1}$, where $T_{0}$ is a closed Fredholm operator on $M$ and $T_{1}$ is a nilpotent operator on $N$. Moreover, $\operatorname{ind}(T)=\kappa\left(T_{0}\right)$. Then we can write

$$
T+\frac{1}{n} I=T_{0}+\frac{1}{n} I_{M} \oplus T_{1}+\frac{1}{n} I_{N} .
$$

From the stability result [7, Theorem 5.22], it follows that $T_{0}+I_{M} / n$ is Fredholm and $\kappa\left(T_{0}+I_{M} / n\right)=\kappa\left(T_{0}\right)$ for all sufficiently large $n$. On the other hand, $T_{1}+I_{N} / n$ is invertible and, thus, $\kappa\left(T_{1}+I_{N} / n\right)=0$. Hence, $T+I / n$ is Fredholm with $\kappa(T+I / n)=$ $\kappa\left(T_{0}\right)=\operatorname{ind}(T)$. Analogously, we can see that the operator $T+F+I / n$ is Fredholm and $\kappa(T+F+I / n)=\operatorname{ind}(T+F)$ for all sufficiently large $n$. Now, since $T+I / n$ is Fredholm and $F$ is a finite-rank operator, it follows that $\kappa(T+(I / n)+F)=\kappa(T+I / n)$. Thus, we have proved that ind $(T+F)=\operatorname{ind}(T)$.

Recall that the ascent of an operator $A$, denoted by $\alpha(A)$, is the smallest non-negative integer $n$ such that $\mathcal{N}\left(A^{n}\right)=\mathcal{N}\left(A^{n+1}\right)$; the descent of $A$, denoted by $\delta(A)$, is the smallest non-negative integer $n$ such that $\mathcal{R}\left(A^{n}\right)=\mathcal{R}\left(A^{n+1}\right)$. A closed operator $A \in \mathcal{C}(H)$ is said to be Drazin invertible if there exists a bounded operator $X \in \mathcal{B}(H)$ with $\mathcal{R}(X) \subseteq \mathcal{D}(A)$ such that

$$
X A X=X, \quad A X u=X A u \quad \text { for all } u \in \mathcal{D}(A)
$$

and

$$
A^{k}(I-A X)=0 \quad \text { for some non-negative integer } k \text {. }
$$


The smallest non-negative integer $k$ that satisfies the preceding equation is called the Drazin index of $A$ and is denoted by $i(A)$. From the theory of the Drazin inverse of closed linear operators $[\mathbf{1 0}]$ we know that an operator $A \in \mathcal{C}(H)$ has a Drazin inverse with $i(A)=k$ if and only if $\alpha(A)=\delta(A)=k$ and $H=\mathcal{R}\left(A^{k}\right) \oplus \mathcal{N}\left(A^{k}\right)$.

Next, using Theorem 2.4 and the characterization of unbounded Fredholm operators given in [11, Theorem 1.1], we characterize unbounded B-Fredholm operators of index 0 as the direct sum of a Drazin invertible closed operator and a finite-rank operator.

Theorem 2.6. Let $T \in \mathcal{C}(H)$ with $\mathcal{D}(T)$ dense in $H$. Then $T$ is B-Fredholm with $d=\operatorname{dis}(T)$ and such that $\operatorname{ind}(T)=0$ if and only if $T=A+F$, where $A$ is a Drazin invertible closed linear operator with Drazin index $i(A)=d, \mathcal{D}(A)=\mathcal{D}(T)$, and $F$ is a finite-rank operator.

Proof. Assume that $T$ is B-Fredholm. From Theorem 2.4 we have $H=M \oplus N$, $N \subseteq \mathcal{N}\left(T^{d}\right), N \nsubseteq \mathcal{N}\left(T^{d-1}\right)$ and, relative to this direct sum, we have the decomposition $T=T_{0} \oplus T_{1}$, where $T_{0}$ is a Fredholm closed operator densely defined on $M$ to $M$ such that $\operatorname{ind}\left(T_{0}\right)=0$ and $T_{1}$ is a nilpotent operator. Now, from [11, Theorem 1.1] it follows that $T_{0}=B+G$, where $B$ is a linear closed operator, $\mathcal{D}(B)=\mathcal{D}\left(T_{0}\right), \mathcal{R}(B)=M$, $\mathcal{N}(B)=\{0\}$ and $G: M \rightarrow M$ is a finite-rank operator. Then $T=\left(B \oplus T_{1}\right)+(G \oplus 0)$. Now define $A=B \oplus T_{1}$ and $F=G \oplus 0$. Clearly, $F: H \rightarrow H$ is a finite-rank operator. We easily see that the operator $A: H \rightarrow H$ is closed with $\mathcal{D}(A)=\mathcal{D}(T)$,

$$
\mathcal{N}\left(A^{d}\right)=\{0\} \oplus \mathcal{N}\left(T_{1}^{d}\right)=\{0\} \oplus N=\mathcal{N}\left(A^{d+1}\right),
$$

$\mathcal{N}\left(A^{d-1}\right) \neq \mathcal{N}\left(A^{d}\right)$ because $N \nsubseteq \mathcal{N}\left(T^{d-1}\right)$, and

$$
\mathcal{R}\left(A^{d}\right)=M \oplus \mathcal{R}\left(T_{1}^{d}\right)=M \oplus\{0\}=\mathcal{R}\left(A^{d+1}\right) .
$$

Hence, $\alpha(A)=\delta(A)=d$ and $H=\mathcal{R}\left(A^{d}\right) \oplus \mathcal{N}\left(A^{d}\right)$. Thus, $A$ is Drazin invertible with $i(A)=d$.

Conversely, let us assume that $T=A+F$, where $A$ is a Drazin invertible closed operator with $\mathcal{D}(A)=\mathcal{D}(T)$ such that $i(A)=d$ and $F$ is a finite-rank operator. Then $A$ is a B-Fredholm operator and $\operatorname{ind}(A)=0$. Thus, by Proposition 2.5 , it follows that $T=A+F$ is a B-Fredholm operator and $\operatorname{ind}(T)=0$.

\section{Properties of B-Fredholm closed operators}

Let $T \in \mathrm{BF}(H)$ and let

$$
\rho_{\mathrm{BF}}(T)=\{\lambda \in \mathbb{C}:(T-\lambda I) \in \mathrm{BF}(H)\}
$$

be the B-Fredholm resolvent of $T$. We denote by $\rho(T)$ the usual resolvent set of $T$.

Theorem 3.1. Let $T \in \mathcal{C}(H)$ be densely defined and such that $\rho(T) \neq \emptyset$. The following conditions are equivalent:

(a) $T$ is B-Fredholm;

(b) $T^{m}$ is B-Fredholm for each $m \geqslant 1$.

Moreover, $\operatorname{ind}\left(T^{m}\right)=m \cdot \operatorname{ind}(T)$. 
Proof. We only need to prove that (a) $\Longrightarrow(\mathrm{b})$. Assume that $T$ is B-Fredholm and let $d=\operatorname{dis}(T)$. Since $T \in \mathrm{q}-\Phi(d)$ and $\rho(T) \neq \emptyset$, from [4, Proposition 2.4] it follows that, for each $m \geqslant 1, T^{m}$ is quasi-Fredholm of degree

$$
d_{m}=\operatorname{dis}\left(T^{m}\right)= \begin{cases}1 & \text { if } m \geqslant d \\ k \text { with }(k-1) m \leqslant d \leqslant k m & \text { if } m \leqslant d .\end{cases}
$$

Suppose that $m \leqslant d$. Let us prove that $\operatorname{dim} \mathcal{N}\left(T^{m}\right) \cap \mathcal{R}\left(T^{k m}\right)<\infty$ and $\operatorname{codim} \mathcal{R}\left(T^{m}\right)+$ $\mathcal{N}\left(T^{k m}\right)<\infty$.

By applying Theorem 2.4, we obtain $T=T_{0} \oplus T_{1}$, where $T_{0}$ is a closed Fredholm operator densely defined on $M, T_{1}$ is a nilpotent operator on $N$ of degree $d$ and $\operatorname{ind}(T)=$ $\kappa\left(T_{0}\right)$. Since $k m \geqslant d$ we obtain $T^{(k+1) m}=T_{0}^{(k+1) m} \oplus 0$. Now, from [5, Theorem IV.2.7] it follows that $T_{0}^{(k+1) m}$ is a Fredholm operator and, consequently, $\operatorname{dim} \mathcal{N}\left(T_{0}^{(k+1) m}\right)<\infty$ for each $m \geqslant 1$. Now, using [6, Lemma 3.1] we get

$$
\begin{aligned}
\operatorname{dim} \mathcal{N}\left(T^{m}\right) \cap \mathcal{R}\left(T^{k m}\right) & =\operatorname{dim} \frac{\mathcal{N}\left(T^{(k+1) m}\right)}{\mathcal{N}\left(T^{k m}\right)} \\
& =\operatorname{dim} \frac{\mathcal{N}\left(T_{0}^{(k+1) m}\right)+N}{\mathcal{N}\left(T_{0}^{k m}\right)+N} \\
& =\operatorname{dim} \frac{\mathcal{N}\left(T_{0}^{(k+1) m}\right)}{\mathcal{N}\left(T_{0}^{k m}\right)}<\infty .
\end{aligned}
$$

On the other hand, since $T^{*}$ is also B-Fredholm and $d=\operatorname{dis}\left(T^{*}\right)$, using an argument similar to that above we can see that

$$
\operatorname{dim} \mathcal{N}\left(T^{* m}\right) \cap \mathcal{R}\left(T^{* k m}\right)<\infty .
$$

It follows from [8, Proposition 3.3.5] that

$$
\operatorname{codim} \mathcal{R}\left(T^{m}\right)+\mathcal{N}\left(T^{k m}\right)=\operatorname{dim} \mathcal{N}\left(T^{* m}\right) \cap \mathcal{R}\left(T^{* k m}\right)<\infty .
$$

Finally, since $T^{m}=T_{0}^{m} \oplus T_{1}^{m}$ with $T_{0}^{m}$ a Fredholm operator densely defined on $M$ and $T_{1}^{m}$ a nilpotent operator on $N$, and from the fact that $\kappa\left(T_{0}^{m}\right)=m \cdot \kappa\left(T_{0}\right)$, we conclude that $\operatorname{ind}\left(T^{m}\right)=\kappa\left(T_{0}^{m}\right)=m \cdot \operatorname{ind}(T)$.

Now we consider the case when $m \geqslant d$. We know that $\operatorname{dis}\left(T^{m}\right)=1$ and $T^{m}=T_{0}^{m} \oplus 0$. Proceeding as in the above case we can prove that $\operatorname{dim} \mathcal{N}\left(T^{m}\right) \cap \mathcal{R}\left(T^{m}\right)<\infty$ and $\operatorname{codim} \mathcal{R}\left(T^{m}\right)+\mathcal{N}\left(T^{m}\right)<\infty$.

Given a polynomial $p(\lambda)=\prod_{i=1}^{n}\left(\lambda-\lambda_{i} I\right)^{m_{i}}$, define $p(T)=\prod_{i=1}^{n}\left(T-\lambda_{i} I\right)^{m_{i}}$. It is well known that $\mathcal{D}(p(T))=\mathcal{D}\left(T^{s}\right)$, where $s=m_{1}+\cdots+m_{n}$ and that if $T$ is closed and densely defined with $\rho(T) \neq \emptyset$, then the operator $p(T)$ is closed and densely defined.

Lemma 3.2. Let $\left\{\lambda_{i}\right\}_{1 \leqslant i \leqslant n}$ be a sequence of distinct complex numbers, let $\left\{m_{i}\right\}_{1 \leqslant i \leqslant n}$ be a sequence of positive integers and let $d$ be a positive integer. Then

$$
\mathcal{N}\left(\prod_{i=1}^{n}\left(T-\lambda_{i} I\right)^{m_{i}}\right) \cap \mathcal{R}\left(\prod_{i=1}^{n}\left(T-\lambda_{i} I\right)^{m_{i} d}\right)=\sum_{i=1}^{n} \mathcal{N}\left(\left(T-\lambda_{i} I\right)^{m_{i}}\right) \cap \mathcal{R}\left(\left(T-\lambda_{i} I\right)^{m_{i} d}\right) .
$$


Proof. We prove the inclusion ' $\subseteq$ ' by induction on $n$. The case when $n=1$ is obvious. Assume that the inclusion is true for $k$ and let

$$
u \in \mathcal{N}\left(\prod_{i=1}^{k+1}\left(T-\lambda_{i} I\right)^{m_{i}}\right) \cap \mathcal{R}\left(\prod_{i=1}^{k+1}\left(T-\lambda_{i} I\right)^{m_{i} d}\right) .
$$

Then

$$
\left(T-\lambda_{k+1} I\right)^{m_{k+1}} u \in \mathcal{N}\left(\prod_{i=1}^{k}\left(T-\lambda_{i} I\right)^{m_{i}}\right) \cap \mathcal{R}\left(\prod_{i=1}^{k}\left(T-\lambda_{i} I\right)^{m_{i} d}\right) .
$$

By the induction assumption, $\left(T-\lambda_{k+1} I\right)^{m_{k+1}} u=\sum_{i=1}^{k} v_{i}$ with $v_{i} \in \mathcal{N}\left(\left(T-\lambda_{i} I\right)^{m_{i}}\right) \cap$ $\mathcal{R}\left(\left(T-\lambda_{i} I\right)^{m_{i} d}\right)$. Now, for any $i, 1 \leqslant i \leqslant k$, since the polynomials $\left(\lambda-\lambda_{i}\right)^{m_{i}}$ and $\left(\lambda-\lambda_{k+1}\right)^{m_{k+1}}$ are relatively prime, there exist polynomials $q_{1 i}(\lambda), q_{2 i}(\lambda)$ such that

$$
q_{1 i}(\lambda)\left(\lambda-\lambda_{i}\right)^{m_{i}}+q_{2 i}(\lambda)\left(\lambda-\lambda_{k+1}\right)^{m_{k+1}}=1 .
$$

Hence, we deduce that if $x \in \mathcal{D}\left(T^{p}\right)$ for sufficiently large $p$, then

$$
q_{1 i}(T)\left(T-\lambda_{i} I\right)^{m_{i}} x+q_{2 i}(T)\left(T-\lambda_{k+1} I\right)^{m_{k+1}} x=x .
$$

Therefore, since $v_{i} \in \mathcal{N}\left(\left(T-\lambda_{i} I\right)^{m_{i}}\right)$ we see that $v_{i}=q_{2 i}(T)\left(T-\lambda_{k+1} I\right)^{m_{k+1}} v_{i}$. Define $w_{i}=q_{2 i}(T) v_{i}$ for all $i, 1 \leqslant i \leqslant k$ and $w_{k+1}=u-\sum_{i=1}^{k} w_{i}$. We observe that

$$
w_{i} \in \mathcal{N}\left(\left(T-\lambda_{i} I\right)^{m_{i}}\right) \cap \mathcal{R}\left(\left(T-\lambda_{i} I\right)^{m_{i} d}\right), \quad 1 \leqslant i \leqslant k .
$$

Moreover,

$$
\left(T-\lambda_{k+1} I\right)^{m_{k+1}} w_{k+1}=\left(T-\lambda_{k+1} I\right)^{m_{k+1}} u-\sum_{i=1}^{k}\left(T-\lambda_{k+1} I\right)^{m_{k+1}} q_{2 i}(T) v_{i} .
$$

Hence,

$$
\left(T-\lambda_{k+1} I\right)^{m_{k+1}} w_{k+1}=\left(T-\lambda_{k+1} I\right)^{m_{k+1}} u-\sum_{i=1}^{k} v_{i}=0 .
$$

Thus, $w_{k+1} \in \mathcal{N}\left(\left(T-\lambda_{k+1} I\right)^{m_{k+1}}\right)$. Since, for all $i, 1 \leqslant i \leqslant k, \mathcal{N}\left(\left(T-\lambda_{i} I\right)^{m_{i}}\right) \subseteq$ $\mathcal{R}\left(\left(T-\lambda_{k+1} I\right)^{m_{k+1} d}\right)$, we see that $w_{i} \in \mathcal{R}\left(\left(T-\lambda_{k+1} I\right)^{m_{k+1} d}\right)$ and so we conclude that $w_{k+1} \in \mathcal{R}\left(\left(T-\lambda_{k+1} I\right)^{m_{k+1} d}\right)$. Thus,

$$
u=\sum_{i=1}^{k+1} w_{i} \in \sum_{i=1}^{k+1} \mathcal{N}\left(\left(T-\lambda_{i} I\right)^{m_{i}}\right) \cap \mathcal{R}\left(\left(T-\lambda_{i} I\right)^{m_{i} d}\right) .
$$

Now we prove the reverse inclusion by induction. The case $n=1$ is obvious. Assume that the inclusion is true for $k$ and let

$$
u \in \sum_{i=1}^{k+1} \mathcal{N}\left(\left(T-\lambda_{i} I\right)^{m_{i}}\right) \cap \mathcal{R}\left(\left(T-\lambda_{i} I\right)^{m_{i} d}\right) .
$$


Then $u=v_{k+1}+w$ with $v_{k+1} \in \mathcal{N}\left(\left(T-\lambda_{k+1} I\right)^{m_{k+1}}\right) \cap \mathcal{R}\left(\left(T-\lambda_{k+1} I\right)^{m_{k+1} d}\right)$ and

$$
w \in \sum_{i=1}^{k} \mathcal{N}\left(\left(T-\lambda_{i} I\right)^{m_{i}}\right) \cap \mathcal{R}\left(\left(T-\lambda_{i} I\right)^{m_{i} d}\right) .
$$

By the induction assumption, we have

$$
w \in \mathcal{N}\left(\prod_{i=1}^{k}\left(T-\lambda_{i} I\right)^{m_{i}}\right) \cap \mathcal{R}\left(\prod_{i=1}^{k}\left(T-\lambda_{i} I\right)^{m_{i} d}\right) .
$$

Hence,

$$
\prod_{i=1}^{k+1}\left(T-\lambda_{i} I\right)^{m_{i}} u=\prod_{i=1}^{k+1}\left(T-\lambda_{i} I\right)^{m_{i}} v_{k+1}+\prod_{i=1}^{k+1}\left(T-\lambda_{i} I\right)^{m_{i}} w=0 .
$$

On the other hand, we can deduce that there exist polynomials $q_{1}(\lambda)$ and $q_{2}(\lambda)$ such that if $x \in \mathcal{D}\left(T^{p}\right)$ for sufficiently large $p$, then

$$
q_{1}(T)\left(T-\lambda_{k+1} I\right)^{m_{k+1} d} x+q_{2}(T) \prod_{i=1}^{k}\left(T-\lambda_{i} I\right)^{m_{i} d} x=x .
$$

We observe that $u \in \mathcal{D}\left(T^{p}\right)$ for all $p \geqslant 0$ and then

$$
u=q_{1}(T)\left(T-\lambda_{k+1} I\right)^{m_{k+1} d} w+q_{2}(T) \prod_{i=1}^{k}\left(T-\lambda_{i} I\right)^{m_{i} d}\left(v_{k+1}+w\right) .
$$

Hence,

$$
u=q_{1}(T)\left(T-\lambda_{k+1} I\right)^{m_{k+1} d} w+q_{2}(T) \prod_{i=1}^{k}\left(T-\lambda_{i} I\right)^{m_{i} d} v_{k+1} .
$$

From this, and the fact that $v_{k+1} \in \mathcal{R}\left(\left(T-\lambda_{k+1} I\right)^{m_{k+1} d}\right)$, we see that

$$
u \in \mathcal{R}\left(\prod_{i=1}^{k+1}\left(T-\lambda_{i} I\right)^{m_{i} d}\right)
$$

Consequently,

$$
u \in \mathcal{N}\left(\prod_{i=1}^{k+1}\left(T-\lambda_{i} I\right)^{m_{i}}\right) \cap \mathcal{R}\left(\prod_{i=1}^{k+1}\left(T-\lambda_{i} I\right)^{m_{i} d}\right)
$$

Theorem 3.3. Let $T \in \mathcal{C}(H)$ with dense domain and such that $\rho(T) \neq \emptyset$, and let $p(\lambda)=\prod_{i=1}^{n}\left(\lambda-\lambda_{i}\right)^{m_{i}}$ be a polynomial of degree $s$ with complex coefficients. Then

$$
0 \in \rho_{\mathrm{BF}}(p(T)) \Longleftrightarrow \lambda_{1}, \ldots, \lambda_{n} \in \rho_{\mathrm{BF}}(T) .
$$

Moreover, in this case $\operatorname{ind}(p(T))=\sum_{i=1}^{n} m_{i} \cdot \operatorname{ind}\left(T-\lambda_{i} I\right)$. 
Proof. Suppose that $p(T)$ is B-Fredholm. We shall prove that $\left(T-\lambda_{i} I\right)$ is B-Fredholm for all $i, 1 \leqslant i \leqslant n$. Since $p(T)$ is quasi-Fredholm, from [4, Theorem 3.1], it follows that $T-\lambda_{i} I$ is quasi-Fredholm for $1 \leqslant i \leqslant n$. Now, using [4, Proposition 2.4], we find that $\left(T-\lambda_{i} I\right)^{m_{i}}$ is quasi-Fredholm for all $i, 1 \leqslant i \leqslant n$. Let $d=\operatorname{dis}(p(T))$ and $d_{i}=\operatorname{dis}\left(\left(T-\lambda_{i} I\right)^{m_{i}}\right)$ for all $i, 1 \leqslant i \leqslant n$. We have

$$
\operatorname{dim} \mathcal{N}(p(T)) \cap \mathcal{R}\left((p(T))^{d}\right)<\infty \quad \text { and } \quad \operatorname{codim} \mathcal{R}(p(T))+\mathcal{N}\left((p(T))^{d}\right)<\infty
$$

because $p(T)$ is B-Fredholm. By applying Lemma 3.2 we obtain

$$
\mathcal{N}(p(T)) \cap \mathcal{R}\left((p(T))^{d}\right)=\sum_{i=1}^{n} \mathcal{N}\left(\left(T-\lambda_{i} I\right)^{m_{i}}\right) \cap \mathcal{R}\left(\left(T-\lambda_{i} I\right)^{m_{i} d}\right) .
$$

Hence, it follows that, for all $i$ with $1 \leqslant i \leqslant n$, we have

$$
\operatorname{dim} \mathcal{N}\left(\left(T-\lambda_{i} I\right)^{m_{i}}\right) \cap \mathcal{R}\left(\left(T-\lambda_{i} I\right)^{m_{i} d}\right)<\infty .
$$

By [4, Lemma 2.2] we have $d_{i}=\operatorname{dis}\left(\left(T-\lambda_{i} I\right)^{m_{i}}\right) \leqslant d$ for all $i, 1 \leqslant i \leqslant n$, and, thus,

$$
\operatorname{dim} \mathcal{N}\left(\left(T-\lambda_{i} I\right)^{m_{i}}\right) \cap \mathcal{R}\left(\left(T-\lambda_{i} I\right)^{m_{i} d_{i}}\right)<\infty .
$$

On the other hand, applying [8, Proposition 3.3.5], we get

$$
\begin{aligned}
\operatorname{codim} \mathcal{R}(p(T))+\mathcal{N}\left((p(T))^{d}\right) & =\operatorname{dim}\left(\mathcal{R}(p(T))+\mathcal{N}\left((p(T))^{d}\right)\right)^{\perp} \\
& =\operatorname{dim} \mathcal{R}\left((p(T))^{* d}\right) \cap \mathcal{N}\left(p(T)^{*}\right)<\infty
\end{aligned}
$$

Now, using the symmetry between the conditions on $p(T)$ and on $p(T)^{*}$, and arguing as above, for $1 \leqslant i \leqslant n$, we obtain

$$
\operatorname{dim} \mathcal{R}\left(\left(T-\lambda_{i} I\right)^{* m_{i} d_{i}}\right) \cap \mathcal{N}\left(\left(T-\lambda_{i} I\right)^{* m_{i}}\right)<\infty .
$$

Hence, we conclude that, for all $1 \leqslant i \leqslant n$,

$$
\operatorname{dim}\left(\mathcal{R}\left(\left(T-\lambda_{i} I\right)^{m_{i}}\right)+\mathcal{N}\left(\left(T-\lambda_{i} I\right)^{m_{i} d_{i}}\right)\right)^{\perp}<\infty .
$$

Thus, we have proved that $\left(T-\lambda_{i} I\right)^{m_{i}}$ is B-Fredholm for $1 \leqslant i \leqslant n$. Using Theorem 3.1, we conclude that $\left(T-\lambda_{i} I\right)$ is B-Fredholm for $1 \leqslant i \leqslant n$. Conversely, suppose that $\left(T-\lambda_{i} I\right)$ is B-Fredholm for all $i, 1 \leqslant i \leqslant n$. Since each $\left(T-\lambda_{i} I\right)$ is quasi-Fredholm, using [4, Theorem 3.1], we conclude that $P(T)$ is quasi-Fredholm. Moreover, if $d_{i}=$ $\operatorname{dis}\left(\left(T-\lambda_{i} I\right)^{m_{i}}\right)$ for $1 \leqslant i \leqslant n$ then $d=\operatorname{dis}(p(T))=\max \left\{d_{i}: 1 \leqslant i \leqslant n\right\}$. If it happens that $\operatorname{dim} \mathcal{N}(p(T)) \cap \mathcal{R}\left(p(T)^{d}\right)=\infty$, then from Lemma 3.2 it would follow that, for some $i, 1 \leqslant i \leqslant n, \operatorname{dim} \mathcal{N}\left(\left(T-\lambda_{i} I\right)^{m_{i}}\right) \cap \mathcal{R}\left(\left(T-\lambda_{i} I\right)^{m_{i} d}\right)=\infty$ and we should arrive at a contradiction of the fact that $\operatorname{dim} \mathcal{N}\left(\left(T-\lambda_{i} I\right)^{m_{i}}\right) \cap \mathcal{R}\left(\left(T-\lambda_{i} I\right)^{m_{i} d_{i}}\right)<\infty$ for all $1 \leqslant i \leqslant n$.

Analogously, if we suppose that $\operatorname{codim} \mathcal{R}(p(T))+\mathcal{N}\left(p(T)^{d}\right)=\infty$, we arrive at a contradiction. 
Now assume that $p(T)$ is B-Fredholm with $d=\operatorname{dis}(p(T))$. Since

$$
\mathcal{N}(p(T)) \cap \mathcal{R}\left((p(T))^{d}\right)=\sum_{i=1}^{n} \mathcal{N}\left(\left(T-\lambda_{i} I\right)^{m_{i}}\right) \cap \mathcal{R}\left(\left(T-\lambda_{i} I\right)^{m_{i} d}\right),
$$

and since the polynomials $\left(\lambda-\lambda_{i}\right)_{1 \leqslant i \leqslant n}^{m_{i}}$ are relatively prime, we have

$$
\operatorname{dim} \mathcal{N}(p(T)) \cap \mathcal{R}\left((p(T))^{d}\right)=\sum_{i=1}^{n} \operatorname{dim} \mathcal{N}\left(\left(T-\lambda_{i} I\right)^{m_{i}}\right) \cap \mathcal{R}\left(\left(T-\lambda_{i} I\right)^{m_{i} d}\right) .
$$

Similarly, we have

$$
\begin{aligned}
\operatorname{codim} \mathcal{R}(p(T))+\mathcal{N}\left((p(T))^{d}\right) & =\operatorname{dim} \mathcal{N}\left(p\left(T^{*}\right)\right) \cap \mathcal{R}\left(\left(p\left(T^{*}\right)\right)^{d}\right) \\
& =\sum_{i=1}^{n} \operatorname{dim} \mathcal{N}\left(\left(T^{*}-\bar{\lambda}_{i} I\right)^{m_{i}}\right) \cap \mathcal{R}\left(\left(T^{*}-\bar{\lambda}_{i} I\right)^{m_{i} d}\right) \\
& =\sum_{i=1}^{n} \operatorname{codim} \mathcal{R}\left(\left(T-\lambda_{i} I\right)^{m_{i}}\right)+\mathcal{N}\left(\left(T-\lambda_{i} I\right)^{m_{i} d}\right) .
\end{aligned}
$$

Therefore, observing that $d_{i}=\operatorname{dis}\left(\left(T-\lambda_{i} I\right)^{m_{i}}\right) \leqslant d$ for all $1 \leqslant i \leqslant n$, we get

$$
\operatorname{ind}(p(T))=\sum_{i=1}^{n} \operatorname{ind}\left(\left(T-\lambda_{i} I\right)^{m_{i}}\right)=\sum_{i=1}^{n} m_{i} \cdot \operatorname{ind}\left(T-\lambda_{i} I\right) .
$$

Acknowledgements. This research was supported by the Agencia Española de Cooperación Internacional (AECI), Project 201/03/P, and PROTARS Project D11/16 Morocco. The authors are grateful to the referee for careful reading of the paper.

\section{References}

1. M. Berkani, On a class of quasi-Fredholm operators, Integ. Eqns Operat. Theory 34 (1999), 244-249.

2. M. Berkani, Index of B-Fredholm operators and generalization of a Weyl theorem, Proc. Am. Math. Soc. 130 (2002), 1717-1723.

3. M. Berkani And D. Medková, A note on the index of B-Fredholm operators, Math. Bohemica 129 (2004), 177-180.

4. M. Berkani And A. OuAhab, Théorème de l'application spectrale pour le spectre essentiel quasi-Fredholm, Proc. Am. Math. Soc. 128 (1997), 763-774.

5. S. GoldBerG, Unbounded linear operators: theory and applications (Dover, New York, 1985).

6. M. A. KaAshozk, Ascent, descent, nullity and defect, a note on a paper by A. E. Taylor, Math. Annalen 172 (1967), 105-115.

7. T. Kato, Perturbation theory for linear operators (Springer, 1995). 
8. J.-P. LABRouse, Les opérateurs quasi Fredholm: une généralisation des opérateurs semi Fredholm, Rend. Circ. Mat. Palermo 29 (1980), 161-258.

9. M. Mbekhta And V. Müller, On the axiomatic theory of spectrum, II, Studia Math. 119 (1996), 129-147.

10. M. Z. NASHED AND Y. ZHAO, The Drazin inverse for singular evolution equations and partial differential operators, World Scientific Series on Applicable Analysis, Volume 1, pp. 441-456 (World Scientific, Singapore, 1992).

11. A. G. RAmm, A characterization of unbounded Fredholm operators, Cubo 5 (2003), 91-95. 